\title{
Adesão à higienização das mãos dos profissionais da saúde em unidade de terapia intensiva neonatal
}

\author{
Adhesion to the hand hygienization \\ of health professionals in a neonatal \\ intensive care unit
}

\author{
Kátia dos Santos Contreiro ${ }^{1}$ (1) \\ Leonardo Bigolin Jantsch ${ }^{2}$ (1) \\ Andrea Moreira Arrué 3 (1) \\ Diúlia Calegari de Oliveira ${ }^{4}$ (1) \\ Danieli Bandeira 5 (1)
}

\begin{abstract}
${ }^{1}$ Faculdade Integrada de Santa Maria (Santa Maria). Rio Grande do Sul, Brasil. katyaslg@hotmail.com ${ }^{2}$ Autor para correspondência. Universidade Federal de Santa Maria (Palmeira da Missões). Rio Grande do Sul, Brasil. leo_jantsch@hotmail.com 3Universidade Federal do Paraná (Curitiba). Paraná, Brasil. andrea.ensp@gmail.com 4,5 Universidade Federal de Santa Maria (Santa Maria). Rio Grande do Sul, Brasil. diuliacoliveira@gmail.com, danielibandeira22@gmail.com
\end{abstract}

\begin{abstract}
RESUMO | OBJETIVO: Avaliar a adesão dos profissionais de saúde quanto à higienização das mãos em um serviço de Terapia Intensiva Neonatal. MÉTODo: Trata-se de uma pesquisa seccional, realizada por meio de um checklist utilizado pelo Serviço de Controle de Infecções Hospitalares para verificar a adesão das equipes multidisciplinares à higienização das mãos. A coleta ocorreu na unidade de Terapia Intensiva Neonatal, em todos os turnos de trabalho. Foram contabilizadas 1096 observações, analisadas sob estatística descritiva. RESULTADOS: A taxa de adesão foi de 55,4\%, destas $83 \%$ com água e sabão e $17 \%$ por fricção com álcool. O momento de maior adesão com água e sabão foi antes e após o contato com o paciente; e a ação com álcool, antes do contato com o paciente. Os fonoaudiólogos obtiveram a taxa mais elevada de higienização das mãos (93,7\%). CONCLUSÃo: Verificou-se baixa taxa de adesão pelos profissionais de saúde à HM, exceto os fonoaudiólogos, especialmente após risco de exposição a fluídos corporais.
\end{abstract}

DESCRITORES: Equipe multiprofissional. Higiene das mãos. Infecção hospitalar. Profissionais de saúde. Unidade de terapia Intensiva Neonatal.

\begin{abstract}
OBJECTIVE: To evaluate the adherence of health professionals regarding hand hygiene in a Neonatal Intensive Care service. METHOD: This is a sectional survey, carried out through a checklist used by the Hospital Infection Control Service to verify the adherence of multidisciplinary teams to hand hygiene. The collection took place in the Neonatal Intensive Care Unit, in all work shifts. 1096 observations were recorded, analyzed using descriptive statistics. RESULTS: The adherence rate was $55.4 \%$, of which $83 \%$ with soap and water and $17 \%$ through rubbing with alcohol. The moment of greatest adherence with soap and water was before and after contact with the patient; and the action with alcohol, before contact with the patient. Speech therapists obtained the highest rate of hand hygiene (93.7\%). CONCLUSION: There was a low rate of adherence by health professionals to $\mathrm{MH}$, except for speech therapists, especially after risk of exposure to body fluids.
\end{abstract}

DESCRIPTORS: Multiprofessional team. Hand hygiene. Hospital infection. Health professionals. Neonatal Intensive Care Unit. 


\section{Introdução}

As infecções relacionadas à assistência à saúde (IRAS), são infecções causadas por microrganismos que advém de diversos sítios corporais de um paciente, entre pacientes e até mesmo entre esses e o âmbito da assistência, em que as mãos dos profissionais de saúde constituem fonte e veículo de transmissão desses germes ${ }^{1}$. Conforme a Organização Mundial de Saúde (OMS), atualmente as IRAS representam uma preocupação de ordem internacional, pois envolvem a atuação dos profissionais de saúde, qualidade dos materiais de uso cotidiano e das instalações físicas hospitalares².

Essas infecções crescem a cada dia no Brasil, representando um problema de saúde pública, devido à alta incidência de infecção em pacientes hospitalizados. Ademais, prolongam a estadia do paciente no momento da internação, resultando em um ônus financeiro tanto para o paciente quanto para os serviços de saúde, bem como a necessidade de tratamento medicamentoso, inúmeros procedimentos, danos sociais e psicológicos aos atores envolvidos ${ }^{3}$.

Pacientes que ficam hospitalizados em Unidade de terapia intensiva (UTI), conforme a gravidade de sua doença, são expostos a procedimentos invasivos e prolongamento da internação, assim possuem alto risco de adquirir infecções ${ }^{4}$. Em terapia Intensiva Neonatal, a realidade não se difere, recém-nascidos graves, além da imaturidade fisiológica, apresentam a exposição ambiental aos diversos fatores de risco para tais infecções ${ }^{5}$.

No Brasil a mortalidade neonatal, representa cerca de $60 \%$ dos índices de mortalidade infantil, e a sepse neonatal é uma das principais causas. As infecções associadas a assistência a saúde afetam mais de $30 \%$ dos neonatos, quando afrontado com a população pediátrica de maior idade, seus índices podem ser até cinco vezes mais elevados. Os principais fatores de risco para o desenvolvimento de infecções neonatais tardias, em neonatos, se dão pela utilização de cateter venoso central, o peso ao nascer e a ventilação mecânica ${ }^{5}$.

Como estratégias de prevenção e minimização das IRAS, práticas simples de higienização das mãos (HM), podem ser adotadas como proposta da redução desses índices. Nessa perspectiva, se faz necessário a sensibilização da equipe profissional de saúde em adotar esta prática como método de profilaxia para o controle destas infecções. O controle das IRAS é um evento multicausal, que não representa apenas em uma ação isolada, mas que envolve todos os indivíduos da equipe, pois estes estão em contato direto e continuo com o paciente ${ }^{6}$.

A higienização das mãos é considerada uma prática segura e vem sendo consolidada desde o final do século XIX em que a lavagem das mãos com água clorada e sabão viesse a evitar a transmissão da febre puerperal, pelas mãos dos profissionais que prestassem cuidados aos pacientes nos serviços de saúde. $\mathrm{Na}$ época essa prática reduziu drasticamente as taxas de mortalidade de $33 \%$ para $2 \%$. Atualmente, designada como lavagem das mãos e definida por qualquer ação de higienizar as mãos (higiene simples, higiene antisséptica e fricção antisséptica das mãos). Para prevenir a transmissão de microrganismo para pacientes e profissionais da saúde, consequentemente, evitando infecções ${ }^{5}$.

A higienização das mãos deve ser realizada antes e após o contato com paciente, antes de realizar procedimentos, antes de calçar as luvas e após a remoção das mesmas ${ }^{8}$. As mãos são meios de transmissão, e mesmo que a técnica seja amplamente reconhecida como meio mais eficaz para prevenir estas infecções, infelizmente percebe-se que a adesão está muito aquém․

Cientificamente, a redução na taxa de infecção está diretamente relacionada ao aumento da higienização das mãos, mesmo com estas evidências, sua adesão tem a taxa média de $40 \%$. Nesse sentido, a OMS desenvolveu estratégia multimodal "uma assistência limpa é uma assistência segura", instigando os serviços de saúde e levando-os a priorizarem a higienização das mãos nas instituições ${ }^{10}$. As estratégias contemplam a educação dos profissionais, monitoração das práticas de higienização das mãos, fixação de lembretes e feedback do desempenho. Esta temática no Brasil é debatida, entretanto, é necessário verificar o impacto das estratégias multimodal de promoção, referentes à higienização das mãos quanto à taxa de adesão. 
Desta forma, visando a diminuição dos índices de infecção em UTI a higienização das mãos busca a prevenção dessas infecções. Além disso, avaliar os índices de adesão, oportunidades e processos que envolvem a higienização das mãos pelos profissionais da saúde, em uma UTI neonatal, impulsiona novos estudos, capacitações e estratégias no intuito de promoção e prevenção da saúde.

Diante do exposto, o presente estudo tem como objetivo avaliar a adesão dos profissionais de saúde quanto à higienização das mãos em um serviço de Terapia Intensiva Neonatal.

\section{Método}

Trata-se de um estudo seccional descritivo realizado em uma Unidade de Terapia Intensiva Neonatal de um hospital universitário de alta complexidade, referência para duas regiões de saúde do interior do Rio Grande do Sul. O serviço é composto por 18 leitos de terapia intensiva e semi-intensiva.

Os dados foram extraídos de um banco de dados que continha as informações coletadas pelo Serviço de Controle de Infecção Hospitalar (SCIH) do referido hospital, cenário de estudo. Os dados foram coletados por rotina do serviço, como estratégia de identificação das taxas de adesão e construção de indicadores de adesão de HM nos serviços de UTI. Os participantes foram observados por intermédio de um instrumento em forma de checklist, elaborado pelo $\mathrm{SCIH}$, utilizado para avaliar a adesão dos profissionais da saúde à $\mathrm{HM}^{11}$. Na investigação foram considerados os seguintes conceitos:

Indicação: Corresponde a momentos precisos durante a assistência ao paciente. ("Meus cinco momentos para $\mathrm{HM}^{\prime \prime}$ ). Momento 1- antes do contato com o paciente; 2 - antes da realização de procedimento asséptico; 3- após risco de exposição a fluídos corporais; 4- após contato com o paciente; 5- após contato com áreas próximas ao paciente ${ }^{11}$.

Ação: Esta é considerada necessária contanto que ela corresponda pelo menos uma indicação. Podendo ser realizada esta ação de duas formas: friccionando as mãos com uma preparação alcoólica ou higienizando as mãos com água e sabonete ${ }^{11}$.

Oportunidade: Desta forma podendo haver várias razões simultâneas para HM, pois a oportunidade é uma unidade que responde a ação, ela determina a necessidade de higienizar as mãos, pela razão simples ou múltipla (a indicação que leva à ação). Onde ela constitui o denominador para aferir a taxa de adesão à HM por profissionais de saúde ${ }^{11}$.

O checklist foi elaborado, pela equipe do $\mathrm{SCIH}$, a partir do manual do Ministério da Saúde (MS) ${ }^{12}$, por meio do qual os profissionais observaram e registravam o número de oportunidades realizadas com indicação nos 5 momentos. A ação foi observada e classificada em: fricção com álcool, lavagem com água e sabão, e se a ação não realizada.

A observação e o preenchimento do checklist foram realizados pela equipe do $\mathrm{SCIH}$, previamente capacitada e seguiu um roteiro pré-estabelecido. A coleta dos dados na UTIN ocorreu no período de junho a agosto de 2016, contemplando 60 dias de observações consecutivos, totalizando três horas de observações, completando uma hora em cada turno de trabalho (manhã, tarde e noite). Cabe destacar que foram dez dias de observação no mês de junho, 25 dias mês de julho e 25 dias de observação mês de agosto de 2016.

Os profissionais de saúde presentes na UTIN, no período citado, em atendimento direto ao paciente foram: enfermeiros (32), fisioterapeutas (5), fonoaudiólogas (2), médicos pediatras/neonatologistas (15), médicos residentes (20), técnicos e auxiliares de enfermagem (50), técnicos em radiologia (3) e outros (assistente social, médicos (outras especialidades), psicólogos) (10).

A análise se deu por meio da estatística descritiva com frequência absoluta e relativa. Utilizou-se o programa Epiinfo versão 6.0.

O estudo teve aprovação institucional e do Comitê de Ética em Pesquisa da Universidade Federal de Santa Maria, por meio da plataforma Brasil, sob número de CAAE: $61165116.4 .0000 .5346 \mathrm{e}$ registo $1.860 .437 \mathrm{em}$ dezembro de 2016. 


\section{Resultados}

Conforme os resultados, durante os meses de julho a agosto, na Unidade de Terapia Intensiva Neonatal, foram observados 1096 oportunidades de higienização das mãos. Desse total, em 607 (55,4\%) os profissionais de saúde realizaram lavagem das mãos com água e sabão ou fricção com álcool, e em 489 (44,6\%) oportunidades a higienização das mãos não foi realizada. A tabela 1 demonstra que houve uma maior adesão a higienização das mãos com água e sabão que a fricção com álcool nos três meses avaliados.

Tabela 1. Taxa de adesão à higienização das mãos pelos profissionais de saúde da Unidade de Terapia Intensiva Neonatal, quanto ao uso de água e sabão, e fricção com o álcool Brasil, 2016

\begin{tabular}{ccccccc}
\hline Mês & \multicolumn{2}{c}{ Adesão } & \multicolumn{2}{c}{ Água e sabão } & \multicolumn{2}{c}{ Álcool } \\
\cline { 2 - 7 } da coleta & $\mathbf{N}$ & $\mathbf{\%}$ & $\mathbf{N}$ & $\mathbf{\%}$ & $\mathbf{N}$ & $\%$ \\
\hline Junho de 2016 & 32 & 44,4 & 20 & 62,5 & 12 & 37,5 \\
Julho de 2016 & 313 & 50,7 & 243 & 77,6 & 70 & 22,4 \\
Agosto de 2016 & 262 & 64,4 & 241 & 92,0 & 21 & 8,0 \\
\hline Total & $\mathbf{6 0 7}$ & $\mathbf{5 5 , 4}$ & $\mathbf{5 0 4}$ & $\mathbf{8 3 , 0}$ & $\mathbf{1 0 3}$ & $\mathbf{1 7 , 0}$ \\
\hline
\end{tabular}

Fonte: Dados do Autor, 2016.

Os momentos com maior taxa de adesão à HM, foram: o Momento 1 (antes do contato com o paciente) com $67,8 \%$ e o Momento 4 (após o contato com o paciente) com 60,9\%. Práticas de higiene com água e sabão foram as mais frequentes em todos os momentos, exceto o momento 5 em que o álcool foi mais utilizado. A menor taxa de adesão à higiene das mãos está no momento 3 (após risco de exposição a fluídos corporais) representando $4,95 \%$ das ocasiões. Esses resultados estão apresentados no gráfico 1.

Gráfico 1. Relação da adesão a higienização das mãos, nos momentos/indicação, pelos profissionais de saúde da Unidade de Terapia Intensiva Neonatal, Santa Maria, Rio Grande do Sul, Brasil, 2016

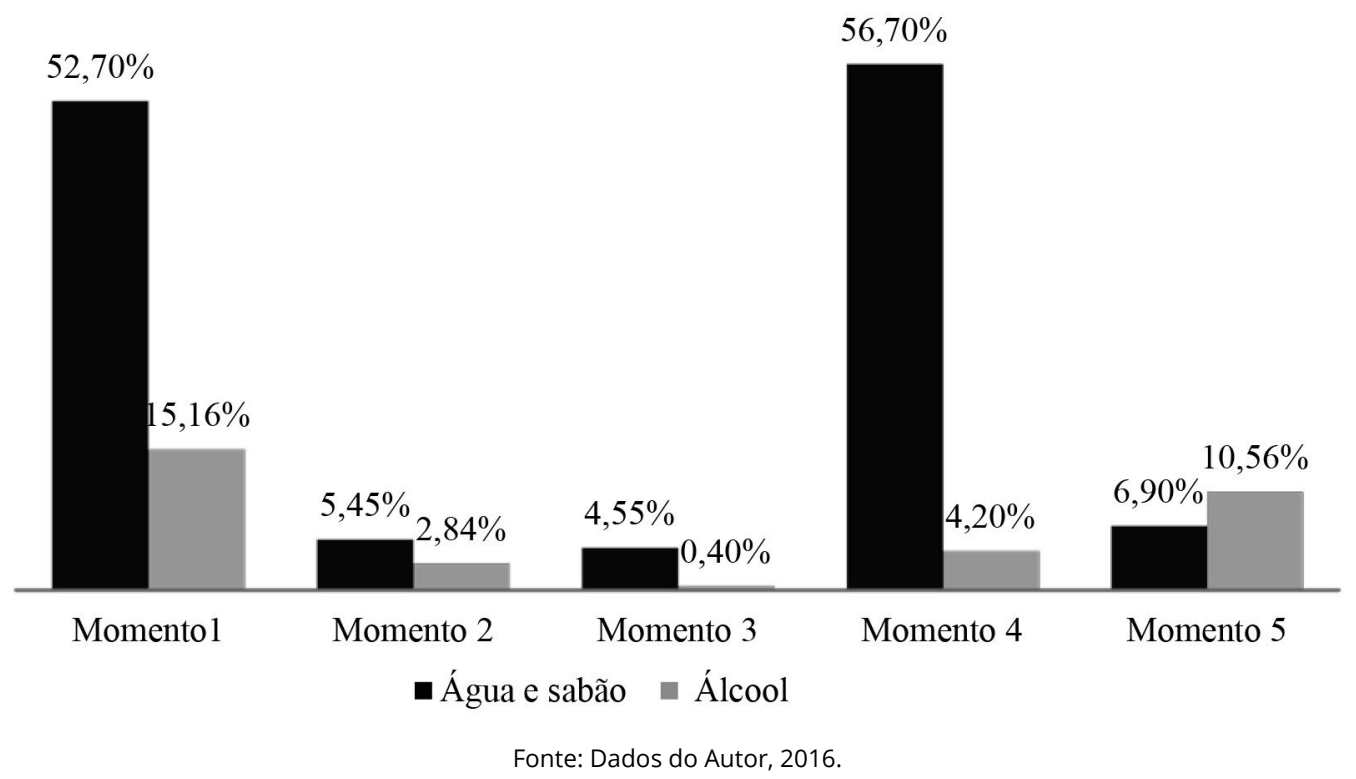


Na tabela 2, destaca-se as taxas de adesão, por oportunidade das categorias profissionais atuantes dentro do cenário de estudo. Aa categorias profissionais com melhores taxas de adesão são os fonoaudiólogos (93,8\%). Cabe destacar ainda que a higiene com água e sabão foi a ação mais utilizada por todas as categorias profissionais.

Tabela 2. Adesão à higienização das mãos por categoria profissional na Unidade de Terapia Intensiva Neonatal. Santa Maria, Rio Grande do Sul, Brasil, 2016

\begin{tabular}{|c|c|c|c|c|}
\hline \multirow{2}{*}{ Categoria profissional } & \multirow{2}{*}{$\begin{array}{l}\text { Oportunidade } \\
\text { (N) }\end{array}$} & \multirow{2}{*}{ Ação } & \multicolumn{2}{|c|}{ Adesão } \\
\hline & & & $\mathbf{n}$ & $\%$ \\
\hline \multirow{3}{*}{ Técnicos de Enfermagem } & \multirow{3}{*}{451} & Água e sabão & 176 & 39,0 \\
\hline & & Fricção com álcool & 47 & 10,4 \\
\hline & & Não realizada & 228 & 50,6 \\
\hline \multirow{3}{*}{ Enfermeiros } & \multirow{3}{*}{371} & Água e sabão & 172 & 46,4 \\
\hline & & Fricção com álcool & 42 & 11,3 \\
\hline & & Não realizada & 158 & 42,6 \\
\hline \multirow{3}{*}{ Fisioterapeutas } & \multirow{3}{*}{101} & Água e sabão & 62 & 61,4 \\
\hline & & Fricção com álcool & 6 & 5,9 \\
\hline & & Não realizada & 33 & 32,7 \\
\hline \multirow{3}{*}{ Médicos } & \multirow{3}{*}{49} & Água e sabão & 32 & 65,3 \\
\hline & & Fricção com álcool & 2 & 4,1 \\
\hline & & Não realizada & 15 & 30,6 \\
\hline \multirow{3}{*}{ Médicos Residentes } & \multirow{3}{*}{23} & Água e sabão & 15 & 65,2 \\
\hline & & Fricção com álcool & 0 & 0,0 \\
\hline & & Não realizada & 8 & 34,8 \\
\hline \multirow{3}{*}{ Fonoaudiólogos } & \multirow{3}{*}{16} & Água e sabão & 15 & 93,8 \\
\hline & & Fricção com álcool & 0 & 0,0 \\
\hline & & Não realizada & 1 & 6,3 \\
\hline \multirow{3}{*}{ Técnicos em Radiologia } & \multirow{3}{*}{3} & Água e sabão & 0 & 0,0 \\
\hline & & Fricção com álcool & 0 & 0,0 \\
\hline & & Não realizada & 3 & 100,0 \\
\hline \multirow{3}{*}{ Outros } & \multirow{3}{*}{76} & Água e sabão & 39 & 51,3 \\
\hline & & Fricção com álcool & 6 & 7,9 \\
\hline & & Não realizada & 43 & 56,6 \\
\hline
\end{tabular}

Fonte: Dados do Autor, 2016.

\section{Discussão}

A higienização das mãos tem um papel central no controle de IRAS, entretanto, os índices de adesão, nesse estudo, atingiram, no máximo, $64 \%$ das oportunidades, no mês de agosto. A taxa de adesão geral à higienização das mãos, na UTIN foi 55,4\% dentre 1096 oportunidades observadas. Pesquisa conduzida em uma UTI, de um hospital em Porto Alegre, realizou 793 observações e a taxa de adesão foi de 43,7\% ${ }^{13}$. Conforme os autores, trata-se de uma estratégia de baixo custo, fácil acesso e eficiente para a prevenção da transmissão de bactérias e IRAS, mas a adesão e o cumprimento das diretrizes ainda são deficientes.

A adesão á HM com água e sabão, nos meses observados foi de $83 \%$ enquanto a fricção com álcool de $17 \%$. Resultado análogo ao estudo brasileiro conduzido em uma UTI neonatal e em uma maternidade sobre HM revelando que 504 (83\%) foram com água e sabão e 103 (17\%) fricção com álcool14. 
Nas últimas décadas, nas UTI, tem se elevado os surtos devido a microrganismos multirresistentes gramnegativos, gerando infecções e assim, acarretando uma ameaça mundial a segurança do paciente e a saúde pública. É pertinente discutir os baixos índices de adesão a HM, visto que esta é considerada como um suporte para segurança do paciente e ferramenta contra a infecção ${ }^{15-16}$.

Observa-se que a adesão foi mais elevada com água e sabão, basicamente, em todos os momentos, exceto no momento 5 (após contato com áreas próximas ao paciente) que se realizou fricção com álcool. No que se refere à adesão geral dos momentos, o estudo caracterizou-se em maior escala no momento 1 (antes do contato com o paciente) com $67,86 \%$ e momento 4 (após o contato com o paciente) com 60,9\%. Em estudo, realizado na Unidade de Terapia Intensiva Neonatal, a adesão dos profissionais aos cinco momentos foi: momento $1(95,1 \%)$, momento $2(75,4 \%)$, momento $3(67,2 \%)$, momento $4(73,8 \%)$ e momento $5(21,3 \%)^{13}$.

Alguns dos fatores que dificultam a adoção dos cinco momentos para a HM nos serviços de saúde envolvem o odor do álcool, por vezes desagradável, e a sensação de mãos pegajosas. Além disso, as preparações alcoólicas contendo aromas podem não ser toleradas por alguns dos profissionais, podendo causar dermatites de contato pela hipersensibilidade ao álcool ou outros aditivos presentes na sua fórmula. Ainda, a utilização de luvas de procedimento com pó é outro fator que implica a não utilização do álcool gel, uma vez que, o pó ao entrar em contato com a preparação alcoólica forma um resíduo indesejável nas mãos ${ }^{17}$.

Outro estudo, em um hospital universitário do estado do Paraná, Brasil, apontou que no que concerne aos cinco momentos de HM para enfermeiros e técnicos de enfermagem, foram observados 158 (24,3\%) momentos antes do contato com o paciente, $35(5,4 \%)$ antes de realizar procedimentos assépticos, 49 (7,5\%) após risco de exposição a fluidos corporais, 247 $(38,0 \%)$ após contato com o paciente e 161 (24,8\%) após contato com as proximidades do paciente, totalizando 650 indicações ${ }^{18}$. Sabe-se que a prática da HM pode comprometer a segurança do paciente, dos profissionais e ambiente próximo. No intuito de desenvolver a técnica correta de HM, a OMS recomenda que as campanhas tragam a correta execução do procedimento nos 5 momentos de HM e o uso de solução alcoólica como padrão ouro ${ }^{11}$.
Sabe-se da importância da correta HM, antes e após o contato com o paciente para prevenir infecções ${ }^{17}$. Perante a esta afirmação, verifica-se que os profissionais observados estão preocupados com a segurança do paciente, pois realizam a HM antes do contato. Estudo ainda destaca que, a adesão espontânea dos profissionais referente a prática de HM nos momentos após o contato com o paciente ou fluidos, estão agregados com autoproteção ${ }^{19}$.

Inquietante a baixa adesão à prática de HM, antes de procedimentos assépticos, que não ocorreu em 95,05\% das oportunidades. O uso de luvas, ao realizar procedimentos assépticos, parece substituir a HM o que pode estar relacionado a déficit de conhecimento acerca da temática ou ao não reconhecimento da importância da sua prática ${ }^{18}$.

Cabe ressaltar que a HM é uma medida individual, simples e menos onerosa para a prevenção das IRAS. Para que a HM seja realizada é fundamental que as instituições de saúde disponham de infraestrutura adequada. Além disso, os profissionais necessitam estar conscientes de sua responsabilidade, sendo influenciadores positivos na prática de $\mathrm{HM}^{20}$. Desta forma, este estudo nos mostra a adesão por categoria profissional, estes resultados são relevantes, pelo fato de que podem ser analisadas as classes profissionais com maior discrepância nos dados.

Com relação à média de não adesão à $\mathrm{HM}$, da equipe multiprofissional, pode-se destacar os técnicos em radiologia (100\%), técnicos em enfermagem $(48,7 \%)$, enfermeiros (45\%) e médicos residentes $(35,4 \%)$. Uma pesquisa evidenciou que a HM foi por vezes ignorada entre $27 \%$ dos médicos e $51,8 \%$ entre técnicos de enfermagem ${ }^{15}$. Destacaram-se os fonoaudiólogos com a taxa mais elevada 93,75\%, divergindo de outras pesquisas que apontam fisioterapeutas, como os profissionais com melhores taxas de adesão ${ }^{14,21}$.

A equipe de enfermagem tem mais proximidade com os pacientes devido aos cuidados prestados, adverso das outras classes profissionais. Vale destacar que, os índices de baixa adesão aos profissionais da enfermagem, pode estar associado, ao maior quantitativo de profissional serem avaliados, e assim tender a uma taxa de adesão menor, bem como, o número de vezes que esses profissionais estão expostos aos momentos de HM. 
É necessário que os profissionais sejam protagonistas na assistência com qualidade e segurança para com os RN, pois estes têm grandes influencias nos cuidados prestados, devem compreender a importância da HM na prevenção e controle de IRAS no âmbito de trabalho.

\section{Conclusão}

Esta pesquisa evidenciou baixa adesão à HM dos profissionais do hospital em estudo. A taxa geral de adesão foi de $55,4 \%$, o momento de HM mais frequente foi nas oportunidades antes e depois do contato com o paciente, destacando-se os profissionais fonoaudiólogos com maior adesão, entre a equipe.

A vigilância da HM é essencial para verificar a adesão a essa técnica e os resultados oportunizam um momento de reflexão ara gestores, líderes e profissionais de saúde quanto às práticas de segurança do paciente. A gestão e o gerenciamento do enfermeiro são fundamentais frente às taxas de baixa adesão.

Discutir a higienização das mãos, se faz necessário e assim, utilizar estratégias de capacitações, feedback, incentivo da equipe e da SCIH são necessárias. Os serviços de saúde devem trabalhar juntos para prover a segurança tanto dos RN quanto dos profissionais. Deve ser esclarecido e pautado a importância dos cinco momentos para a higienização das mãos no controle de IRAS.

\section{Contribuições dos autores}

Contreiro KS, Jantsch LB participaram da concepção, delineamento, busca e análise estatística dos dados da pesquisa, interpretação dos resultados, redação do artigo científico. Arrué AM, participou da concepção, delineamento, análise estatística dos dados da pesquisa, interpretação dos resultados e redação. Oliveira DC, participou da concepção e redação. Bandeira $D$, participou da concepção, interpretação dos resultados e redação.

\section{Conflitos de interesses}

Nenhum conflito financeiro, legal ou político envolvendo terceiros (governo, empresas e fundações privadas, etc.) foi declarado para nenhum aspecto do trabalho submetido (incluindo, mas não se limitando a subvenções e financiamentos, participação em conselho consultivo, desenho de estudo, preparação de manuscrito, análise estatística, etc.).

\section{Referências}

1. Costa M, Rodrigues GMC, Gomes WM, Rezende Junior AA, Cardoso FMN. Principais micro-organismos responsáveis por infecções relacionadas à assistência em saúde (IRAS) me UTIS: uma revisão integrativa. Rev Eletron Facul Evang Ceres. 2019;8(1):06-20. http://dx.doi.org/10.36607/refacer.v8i1.4480

2. Organização Mundial da Saúde (OMS). Salve Vidas: Higienize suas Mãos/ Organização Mundial da Saúde. Guia para a Implementação da Estratégia Multimodal da OMS para a Melhoria da Higiene das Mãos. [Internet]. 2009. Disponível em: https:// proqualis.net/sites/proqualis.net/files/Guia_de_Implementao_ estratgia_multimodal_de_melhoria_da_HM_0.pdf

3. Pereira FGF, Chagas ANS, Freitas MMC, Barros LM, Caetano JA. Caracterização das infecções relacinadas à assistência à saúde em uma unidade de terapia intensiva. Rev Vigil Sanit Debate. 2016;4(1):70-77. http://dx.doi.org/10.3395/2317-269x.00614

4. Carapina JC, Araújo BT, Esteves AC, Pereira DCR. Perfil de suscetibilidade para infecções relacionadas à assistência a saúde (IRAS) no Brasil: uma revisão sistemática. Encontro da rede distrital de avaliação de tecnologias em saúde - Arca Repositório Institucional da Fiocruz [Internet]. 2018. [acesso em 2020 mai 19]. Disponível em: https://www.arca.fiocruz.br/handle/icict/39136

5. Ministério da Saúde. Agência Nacional de Vigilância Sanitária. Série Segurança do Paciente e Qualidade em Serviços de Saúde. Critérios diagnósticos de infecção Associada à assistência à saúde - Neonatologia. [Internet]. 2017. [acesso em 2020 mai 19]. Disponível em: https://www.gov.br/anvisa/pt-br

6. Moutinho AF, Brito ALD, Pinheiro TXA. A infecção relacionada à assistência à saúde na UTI neonatal da maternidade referência em alto risco do Rio Grande do Norte: um desafio aos gestores institucionais. Tempus Actas de Saúde Colet. 2016;10(3):09-17. http://dx.doi.org/10.18569/tempus.v10i3.1540

7. Leuthier RM, Carvalho VMJF, Andrade DEM, Albuquerque KF, Batista TPA, Oliveira MA. Passo a passo na implantação do protocolo de higiene das mãos. Inter Scientia. 2018;6(2):67-78. http://dx.doi.org/10.26843/interscientia.v6i2.660

8. Rock C, Harris AD, Reich NG, Johnson JK, Thom KA. Is hand hygiene before putting on nonsterile gloves in the intensive care unit a waste of health care worker time? A randomized controlled trial. Am J Infect Control. 2015;41(11):994-96. http://dx.doi. org/10.1016/j.ajic.2013.04.007

9. Sunkesula VCK, Meranda D, Kundrapu S, Zabarsky TF, McKee M, Macinga DR, et al. Comparison of hand hygiene monitoring using the 5 moments for hand hygiene method versus a wash in- wash out method. Am J Infect Control. 2015;43(1):16-9. http://dx.doi. org/10.1016/ j.ajic.2014.10.003

10. Prado MF, Oliveira ACJ, Nascimento TMB, Mello WA, Prado DB. Estratégia de promoção a higienização das mãos em unidade de terapia intensiva. Cienc Cuid Saude. 2012;11(3):557-564. http:// dx.doi.org/10.4025/cienccuidsaude.v11i3.16366 
11. Organização Pan-Americana da Saúde (OPAS). Agência Nacional de Vigilância Sanitária. Manual para Observadores: estratégia multimodal da OMS para a melhoria da higienização das mãos. [Internet]. 2008. [acesso em 2020 mai 10]. Disponível em: https://www.paho.org/bra/index.php?option=com_ docman\&view=download\&alias=497-manual-para-observadores7\&category_slug=seguranca-do-paciente-970\&ltemid=965

12. Ministério da Saúde. Portaria 2.616, de 12 de maio de 1998. Dispõe a respeito do controle de infecção hospitalar. Diário Oficial da União. 1998 mai.12.

13. Silva APB, Jericó MC, Rodrigues ML, Rangel RMC, Faria Junior GM, Pereira FR. Adesão da equipe de enfermagem na higienização das mãos em unidade de terapia intensiva neonatal e pediátrica. Arq Ciênc Saúde. 2017;24(1):35-40. http://dx.doi. org/10.17696/2318-3691.24.1.2017.555

14. Souza LM, Ramos MF, Becker ESS, Meirelles LCS, Monteiro SAO. Adesão dos profissionais de terapia intensiva aos cinco momentos da higienização das mãos. Rev Gaúcha Enferm. 2015;36(4):21-8. http://dx.doi.org/10.1590/19831447.2015.04.49090

15. Silva DS, Dourado AMG, Cerqueira CRE, Romero FH, Amaral NA, Pearce PF, et al. Hand hygiene adherence according to World Health Organization Recommendations in a Neonatal Intensive Care Unit. Rev Bras Saude Mater Infant. 2017;17(3):551-559. http://dx.doi.org/10.1590/1806-93042017000300008

16. Rousham EK. Hand hygiene infrastructure and behaviours in resource-limited healthcare facilities. J Hosp Infect. 2016;94(3):284-5. http://dx.doi.org/10.1016/j.jhin.2016.08.015
17. Agência Nacional de Vigilância Sanitária. Nota técnica n01/2018 GVIMS/GGTES/ANVISA: orientações gerais para higiene das mãos em serviços de saúde. [Internet]. 2018. [acesso em 2020 mai 10]. Disponível em: https://www20.anvisa.gov.br/ segurancadopaciente/index.php/publicacoes/item/nota-tecnica-n01-2018-gvims-ggtes-anvisa-orientacoes-gerais-para-higiene-dasmaos-em-servicos-de-saude-2

18. Vasconcelos RO, Alves DCl, Fernandes LM, Oliveira JLC. Adhesión a la higiene de las manos por el equipo de enfermería en la unidad de cuidados intensivos. Enferm Glob. 2018;17(50):430-45. http://dx.doi.org/10.6018/ eglobal.17.2.284131

19. Rodriguez V, Giuffre C, Villa S, Almada G, Prasopa-Palizier $\mathrm{N}$, Gogna $\mathrm{M}$, et al. A multimodal intervention to improve hand hygiene in ICUs in Buenos Aires, Argentina: a stepped wedge trial. Inter J Qual Health Care. 2015;27(5):405-11. http://dx.doi. org/10.1093/intqhe/mzv065

20. Magnago TSBS, Ongaro JD, Greco PBT, Lanes TC, Zottele C, Gonçalves NG, et al. Infraestrutura para higienização das mãos em um hospital universitário. Rev Gaucha Enfer. 2019;40(esp):3-7. http://dx.doi.org//10.1590/1983-1447.2019.20180193

21. Trannin KPP, Campanharo CRV, Lopes MCBT, Okuno MFP, Batista REA. Adesão à higiene das mãos: intervenção e avaliação. Cogitare Enferm. 2016;21(2):01-07. http://dx.doi.org/10.5380/ ce.v2li2.44246 